\title{
Diagnostik und Inklusion
}

\author{
Yvonne Blumenthal \\ Universität Rostock \\ Kathrin Mahlau \\ Universität Greifswald
}

Diagnostik und planvolles pädagogisches Handeln sind seit jeher eng miteinander verknüpft (Ingenkamp/Lissmann 2008). Ganz allgemein lässt sich pädagogische Diagnostik und deren Hauptaufgabe im Sinne Klauers (1982) als „das Insgesamt von Erkenntnisbemühungen im Dienste aktueller pädagogischer Entscheidungen" (ebd., 5) kennzeichnen, das sich im Kontext einer sonderpädagogischen Diagnostik auf die Personengruppe der Kinder mit sonderpädagogischem Förderbedarf bezieht (Bundschuh 2005). Zu den Zielen sonderpädagogischer Diagnostik zählen Maßnahmen der Förderung, Modifikation, Platzierung und Selektion. Vor dem Hintergrund der aktuellen inklusiv ausgerichteten Veränderungen werden sowohl die Personengruppen als auch die Ziele erneut diskutiert.

Im Kontext des bestehenden mehrgliedrigen Schul- und Fördersystems erhalten v. a. Selektionsentscheidungen einen hohen Stellenwert. Dieses Schulsystem befindet sich jedoch seit der Ratifizierung der Behindertenrechtskonvention im Jahr 2009 im Umbruch. Mit dem Abbau separierender Förderorte verlieren Platzierungs- und Selektionsentscheidungen ihre Bedeutung, dagegen rücken für den Unterricht und die Förderung verwertbare Informationen über den Lern- und Entwicklungsprozess eines Kindes in den Mittelpunkt diagnostischer Tätigkeiten. Diese als „formative assessment" (Scriven 1967) bezeichnete diagnostische Strategie stellt ein zentrales Element für passgenaue und flexible Förderentscheidungen dar und wird in Ländern mit inklusiven Schulsystemen (z. B. den USA) v. a. für Kinder mit Lern-, Verhaltens- und Sprachauffälligkeiten seit Jahrzehnten praktiziert. Im Gegensatz zu summativer Diagnostik ist die im deutschsprachigen Raum häufig auch als Lernverlaufsdiagnostik (Klauer 2011) bezeichnete formative Diagnostik durch regelmäßige, hochfrequente Messungen gekennzeichnet. Diese dokumentieren die intraindividuelle Entwicklung während eines Lernprozesses und bilden die Grundlage für die Evaluation des bisherigen pädagogischen Angebots und dessen Optimierung (ein Gedanke, der bereits im Konzept der Förderdiagnostik formuliert wurde). Im Kontext präventiv ausgerichteten pädagogischen Handelns sollte Verlaufsdiagnostik zunächst an alle Kinder einer Lerngruppe adressiert sein, um Besonderheiten in Lernverläufen frühzeitig zu bemerken, angemessen darauf zu reagieren und so eine Förderung von Anfang an zu gewährleisten.

Damit drängen sich pragmatisch assoziierte Fragen auf, z. B.: Wer übernimmt in einem inklusiven Schulsystem welche diagnostischen Aufgaben? Versteht man die Klassenlehrkraft als zuständig für alle Kinder einer Lerngruppe und die sonderpädagogischen Lehrkräfte als Fachkräfte für Kinder mit spezifischen Problemstellungen, dann lässt sich die Aufgabenverteilung nach der Zielgruppe organisieren. Die damit einhergehende Teilung der diagnostischen Kompetenz steht keinesfalls im Wider- 
spruch zu einer gemeinsamen Betrachtung der individuellen Entwicklungsschritte eines Kindes im Rahmen multiprofessioneller Teamberatungen. Durch den Einbezug verschiedener diagnostischer Quellen und Methoden ist vielmehr ein Zugewinn im Hinblick auf die Begleitung, Reflektion und den Entscheidungsprozess für weitere pädagogische Handlungsschritte zu erwarten.

Verlaufsdiagnostik als Ausgangspunkt für eine individualisierte und spezifische Anpassung der Lern- und Förderangebote birgt große Chancen, das wait-to-fail-Prinzip (Hartmann/ Müller 2009) sowie das Etiketten-RessourcenDilemma (Wocken 1996) zu überwinden. Die aktuelle Praxis zeigt jedoch, dass die Zuweisung zeitlicher, personeller und materieller Ressourcen noch an das Konzept der historisch gewachsenen kategorialen Feststellungsdiagnostik gebunden ist. Hier besteht ein zentraler Widerspruch zu schulischer Inklusion, die auf eine sonderpädagogische Grundversorgung angewiesen ist.

Neben der Ressourcenproblematik wird der Aspekt der Kategorisierung bzw. Etikettierung von Menschen mit Behinderungen diskutiert. So stellt Wocken (2011) die Forderung nach einer Dekategorisierung, also nach dem Verzicht einer Zuordnung mittels Etikettierung. Diese sei im inklusiven Unterricht überflüssig, da die Verschiedenheit der Kinder die Voraussetzung inklusiver Beschulung ist und allein die Etikettierung ungünstige soziale Unterschiede provoziere. Heimlich $(2014,16)$ formuliert in diesem Zusammenhang: „Wer mit dem Anspruch der Individualisierung des Bildungsangebotes für alle Kinder in inklusiven Schulen allerdings ernst machen will, der wird auch nicht umhin können, die individuellen Lernund Förderbedürfnisse aller Kinder und Jugendlichen differenziert zu diagnostizieren und entsprechend individuelle Förderpläne für alle zu erstellen. “Ahrbeck (2014) und Preuß-Lausitz (2016) vermuten, dass Kinder mit Förderbedar- fen keine besondere Beachtung im Unterricht erhalten, wenn ihre besonderen Entwicklungslagen nicht explizit herausgestellt werden. Eine Dekategorisierung erschwert zudem die fachliche Kommunikation in professionellen Teams und hätte ein Sinken pädagogischer Qualität zur Folge. Eine fachwissenschaftlich differenzierte Betrachtung dieser Frage vor dem Hintergrund unterschiedlicher sonderpädagogisch relevanter Zielgruppen steht derzeit noch aus.

Zusammenfassend ist festzuhalten, dass innerhalb der (sonder)pädagogischen Diagnostik im inklusiven Schulsystem vor allem eine Fokusverschiebung in Richtung förderrelevanter Prozessdiagnostik und eine Abkehr von bisherigen Zielen wie Selektion und Platzierung stattfindet. Eine Möglichkeit, dies zu realisieren, stellt der Einsatz von Verfahren zur Verlaufsdiagnostik dar. In den letzten Jahren wurden Forschungsaktivitäten zur Entwicklung von curriculumbasierten Verfahren zur Lernverlaufsdiagnostik intensiviert. Da der bloße Einsatz der Verlaufsdiagnostik den Mehrwert einer passgenauen Unterrichts- und Förderplanung nicht automatisch liefert, sondern vielmehr als diagnostisches Mittel fungiert, sollte der Schwerpunkt weiterführender Forschungsbemühungen die professionelle und förderorientierte Verwertung dieser diagnostisch gewonnenen Informationen über das Kind ins Zentrum rücken. Bildungspolitisch wünschenswerte Entscheidungen wie die Einführung einer förderpädagogischen Grundversorgung für alle Schülerinnen und Schüler würden überdies nicht nur die Gewichtung einer Feststellungsdiagnostik verringern, sondern auch die notwendige Überwindung von nicht zu verantwortenden wait-to-fail-Strategien unterstützen.

\section{Literatur}

Ahrbeck, B. (2014): Inklusion. Eine Kritik. Stuttgart: Kohlhammer 
Bundschuh, K. (2005): Einführung in die sonderpädagogische Diagnostik. München: Reinhardt

Hartmann, E.; Müller, C. M. (2008): Schulweite Prävention von Lernproblemen im RTI-Modell. In: Schweizerische Zeitschrift für Heilpädagogik 15 (9), 25-33

Heimlich, U. (2014): Einleitung: Inklusion und Sonderpädagogik. In: Heimlich, U.; Kahlert, J. (Hrsg.): Inklusion in Schule und Unterricht. 2. Aufl. Stuttgart: Kohlhammer, 9-26

Ingenkamp, K.; Lissmann, U. (2008): Lehrbuch der Pädagogischen Diagnostik. Weinheim: Beltz

Klauer, K.J. (1982): Perspektiven pädagogischer Diagnostik. In: Klauer, K.J. (Hrsg.): Handbuch der Pädagogischen Diagnostik, Bd. 1. Düsseldorf: Schwann, 3-14

Klauer, K.J. (2011): Lernverlaufsdiagnostik - Konzept, Schwierigkeiten und Möglichkeiten. In: Empirische Sonderpädagogik 3, 207-224

Preuß-Lausitz, U. (2016): Throughput instead of input. Herausforderungen beim Wegfall der Feststellungsdiagnostik in den Förderbereichen Lernen, Emotionale und soziale Entwicklung und Sprache. In: Zeitschrift für Heilpädagogik 67, 204-214

Scriven, M. (1967): The methodology of evaluation. In: Tyler, R.W.; Gagne, R.M.; Scriven, M. (eds.): Perspectives of Curriculum Evaluation. Chicago, IL: Rand McNally, 39-83
Wocken, H. (1996): Sonderpädagogischer Förderbedarf als systemischer Begriff. In: Sonderpädagogik 26, 34-38

Wocken, H. (2011): Rettet die Sonderschulen? Rettet die Menschenrechte! Ein Appell zu einem differenzierten Diskurs über Dekategorisierung. In: Zeitschrift für Inklusion-online.net 4-2011. Online: http://www.inklusion-online.net/ index.php/inklusion-online/article/view/81/81, 20.6.2017

\section{Anschrift der Autorinnen}

\section{Dr. phil. habil. Kathrin Mahlau}

Ernst-Moritz-Arndt-Universität Greifswald Institut für Erziehungswissenschaften Lehrstuhl für Sonderpädagogik und Inklusion Franz-Mehring-Straße 47

D-17487 Greifswald

E-Mail: kathrin.mahlau@uni-greifswald.de

\section{Dr. Yvonne Blumenthal}

Universität Rostock

Institut für Sonderpädagogische

Entwicklungsförderung und Rehabilitation

Albert-Einstein-Straße 21

D-18059 Rostock

E-Mail: yvonne.blumenthal2@uni-rostock.de 